\title{
Frequency analysis of the magnetization dynamics in thin ellipsoidal magnetic elements
}

\author{
L. Le Guyader,* C. Anceau, A. Kirilyuk, and Th. Rasing \\ IMM, Radboud University Nijmegen, Toernooiveld 1, 6525ED Nijmegen, The Netherlands \\ D. Berkov \\ INNOVENT e.V., Pruessingstrasse 27B, D-07445 Jena, Germany \\ L. Bär \\ SIEMENS AG/CT MM 1, Paul-Gossen-Strasse 100, D-91052 Erlangen, Germany
}

(Received 25 November 2005; published 10 February 2006)

\begin{abstract}
We report a full analysis of the magnetization dynamics in thin ellipsoidal Permalloy $\left(\mathrm{Ni}_{80} \mathrm{Fe}_{20}\right)$ elements, made both experimentally, using an all optical pump-probe scheme based on the magneto-optical Kerr effect, and numerically, using micromagnetic simulations. We have found experimentally as well as numerically that the precession frequencies shift with the aspect ratio of the element, with stronger effects for smaller sizes. Moreover, the magnetization dynamics is found to be inhomogeneous, and can be decomposed into three different kinds of localized precession modes: a low frequency edge mode, a main mode which is composed of several modes with frequencies close to each other, and some higher-order modes.
\end{abstract}

DOI: 10.1103/PhysRevB.73.060402

PACS number(s): 75.70.-i, 75.30.Ds, 76.50.+g, 75.40.Gb

The fastest way so far to reverse the magnetization of a magnetic bit in magnetic random access memory (MRAM) devices is by precessional switching. ${ }^{1-5}$ In practice, this is done by pulling the magnetization of a thin magnetic element out of plane with a short magnetic field pulse. The thus created strong demagnetizing field will drive the magnetization to the reversed position, at which time the magnetic field pulse should be precisely switched off. This will stop the magnetization exactly in the reversed direction without any further oscillations, so-called ringing, that would otherwise slow down the overall reversal process. ${ }^{6-10}$ Severals groups have studied the magnetization dynamics in thin patterned elements,,${ }^{11,12}$ including the effects of defects and the resulting additional damping, ${ }^{13}$ the localized precession modes that may exist in such elements, ${ }^{14-17}$ and the approach for reducing the crosstalk between neighboring bits. ${ }^{18,19}$ A particular problem is how to design an optimal field pulse to switch an element within half a precession period and then stopping the magnetization without any ringing. In practical cases, however, this ringing cannot be completely avoided, because the magnetization dynamics is not homogeneous in most element shapes, so the proper magnetic field pulse varies across an element.

In order to address these problems, we have carried out time-resolved experiments of the precessional magnetization dynamics in thin elliptical Permalloy elements, and verified these data with micromagnetic simulations. We have found that the aspect ratio of the magnetic elements influences both the main precession frequency as well as its overtones. Micromagnetic simulations confirmed the shift of the main frequency to be due to the change in the shape anisotropy. The appearance of several other harmonics, both in the experiments and in the simulations, can be attributed to the deviation of the element shape from a true three-dimensional ellipsoid.

The dynamics of the magnetization vector $M$ under the influence of an effective magnetic field $H_{\text {eff }}$ can be described by the Landau-Lifshitz-Gilbert (LLG) equation, ${ }^{20,21}$

$$
\frac{d M}{d t}=-|\gamma| M \times H_{e f f}+\frac{\alpha}{M_{s}} M \times \frac{d M}{d t} .
$$

For this magnetization dynamics to be homogeneous and to avoid ringing, the effective field $H_{\text {eff }}$ has to be homogeneous across the magnetic element.

For a homogeneous magnetization configuration, this effective field $H_{\text {eff }}$ can be written as

$$
H_{\text {eff }}=H_{\text {ext }}+H_{\text {ani }}+H_{\text {dem }},
$$

with $H_{e x t}$ the external applied field, composed of a bias field $H_{d c}$ and a pulse field $H_{p}, H_{a n i}$ the anisotropy field, and $H_{d e m}$ the demagnetizing field. Because $H_{e x t}$ and $H_{a n i}$ are most likely to be homogeneous over the element, inhomogeneities are mostly related to $H_{d e m}$, which, in turn, is directly dependent on the element shape. In fact, for most shapes the demagnetizing field $H_{d e m}$ is inhomogeneous, with the only exception of a three-dimensional ellipsoid. ${ }^{22}$

In practice, however, a three-dimensional ellipsoid is difficult to realize, the best alternative being a two-dimensional thin ellipsoid. In this paper, we have investigated the precession dynamics in thin elliptical Permalloy elements, with different shapes and sizes ranging from $32 \times 8 \mu \mathrm{m}$ to $4 \times 4 \mu \mathrm{m}$ latteral size and $8 \mathrm{~nm}$ thickness.

The complete sample design, shown in Fig. 1, is close to

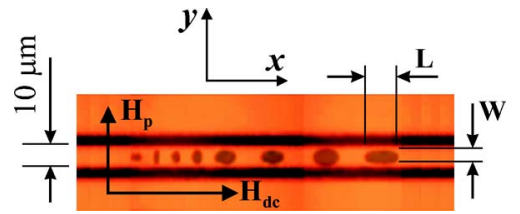

FIG. 1. (Color online) A photograph of the waveguide structure with a series of magnetic elements with different shapes. 


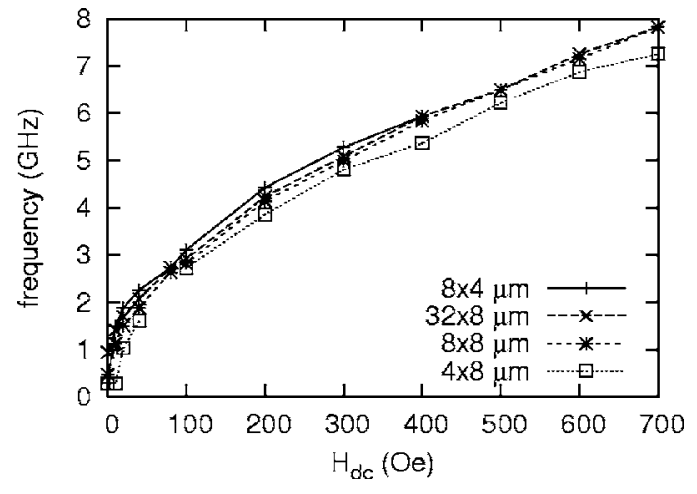

FIG. 2. Precession frequency as function of the applied field $H_{d c}$, for a pulse field $H_{p}$ of $90 \mathrm{Oe}$.

a realistic MRAM device, as the magnetic elements are deposited on top of a strip line. The special part of our design is the photoconductive switch with which we create the pulse field $H_{p}$ by illuminating it with a short and intense $100 \mathrm{fs}$ pump laser pulse. ${ }^{8}$ The typical characteristics of the created field pulse are a rise time of about $10 \mathrm{ps,} \mathrm{a} \mathrm{decay} \mathrm{time} \mathrm{of}$ about 200 ps and a maximum amplitude of 90 Oe. The magnetic elements are deposited using rf sputtering and lithography. The measurements of the magnetization dynamics are performed with an all optical pump-probe technique. The low intensity probe pulse is delayed with respect to the pump laser pulse via an optical delay line and focused on the magnetic element with a microscope objective of N.A. $=0.9$ and $100 \times$ magnification, resulting in a spot size of about $2 \mu \mathrm{m}$ diameter. The polar Kerr rotation of the polarization of the reflected light, which is proportional to the out-of-plane component of the magnetization $M_{z}$, is detected by a balanced photodiodes scheme and lock-in technique. ${ }^{23}$

Figure 2 presents the measured precession frequency as a function of the external applied field $H_{d c}$, for different sizes and shapes of the elements. This figure shows that the elements with a larger length than width $(L>W)$, as defined in Fig. 1, such as $8 \times 4 \mu \mathrm{m}$, have a higher precession frequency than elements with $L<W$, such as $4 \times 8 \mu \mathrm{m}$. This effect is less visible for larger elements, such as $32 \times 8 \mu \mathrm{m}$.

Micromagnetic simulations ${ }^{24}$ were done for elements with similar aspect ratios but smaller size, like $800 \times 400 \times 8 \mathrm{~nm}$, and two and four times larger ones, in order to limit the computation times. We have thus simulated elements which are 10, 5, and 2.5 times smaller than the smallest measured one. The parameters used for the simulation were $4 \pi M_{s}$ $=10807 \mathrm{Oe}$, average crystallite size of $10 \mathrm{~nm}$ with an anisotropy constant $K_{1}=5000 \mathrm{erg} / \mathrm{cm}^{3}$, exchange stiffness $A$ $=10^{-6} \mathrm{erg} / \mathrm{cm}$, which are typical values for sputtered Permalloy films. ${ }^{25}$ The pulse field $H_{p}$ was 45 Oe with a rise time of $10 \mathrm{ps}$, a flat top at 45 Oe during $10 \mathrm{ps}$, and then a decay of 180 ps. The external applied field $H_{d c}$ was 300 Oe.

The results of the simulations are presented in Fig. 3, together with the results of the measurements on the smallest elements. This figure shows that the same shift in the precession frequency occurs, and that this shift becomes larger for smaller elements, in good agreement with the measurements. One should also note that the precession frequency of a circular-shaped element $(L=W)$ is not really affected by its size in the range studied.

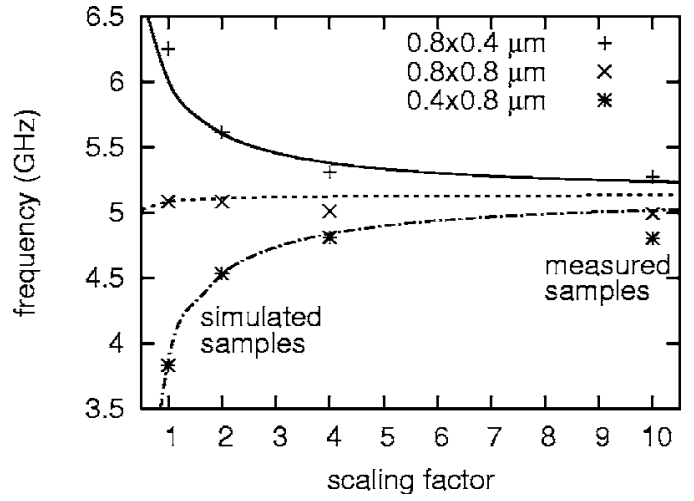

FIG. 3. Precession frequency as function of element size for a given aspect ratio. The points called " $0.8 \times 0.4 \mu \mathrm{m}$ " give the results for this aspect ratio. The real size is given by the scaling factor, so that a scaling factor of 1 corresponds to an element size of 0.8 $\times 0.4 \mu \mathrm{m}$. For a scaling factor of 2 , the size is $1.6 \times 0.8 \mu \mathrm{m}$ and so on. Scaling factor 1,2 , and 4 correspond to simulations, and 10 to measurements. Other aspect ratios are given by the two other kinds of points. The lines correspond to the theoretical curve, given by Eq. (3). Thickness is always $8 \mathrm{~nm}$. The applied field $H_{d c}$ was 300 Oe.

This higher precession frequency for elements with $L>W$ can be fully related to the demagnetizing field $H_{d e m}$. The component of $H_{\text {dem }}$ that compensates the external applied field $H_{d c}$ is $-N_{x} 4 \pi M_{x}$, where $N_{x}$ and $M_{x}$ are the demagnetizing coefficient and component of the magnetization $M$, respectively, in the $x$ direction. As $N_{x}$ is smaller in the case of $L>W$ than $L<W$, the effective field will be higher in that case, resulting in a higher precession frequency for elements with $L>W$ than for elements with $L<W$. Similarly, Fig. 4 shows that the excitation amplitude is larger for the elements with $L<W$. This can also be understood with the help of $H_{d e m}$. At the beginning of the excitation, the magnetization starts to move towards the pulse field $H_{p}$, resulting in a component of $H_{d e m}$ which is $-N_{y} 4 \pi M_{y}$, where $N_{y}$ and $M_{y}$ are the demagnetizing coefficient and magnetization component, respectively, in the $y$ direction. This component of $H_{d e m}$ will partly compensate the pulse field. As $N_{y}$ is smaller in the case of $L<W$ than for $L>W$, the effective pulse field will be

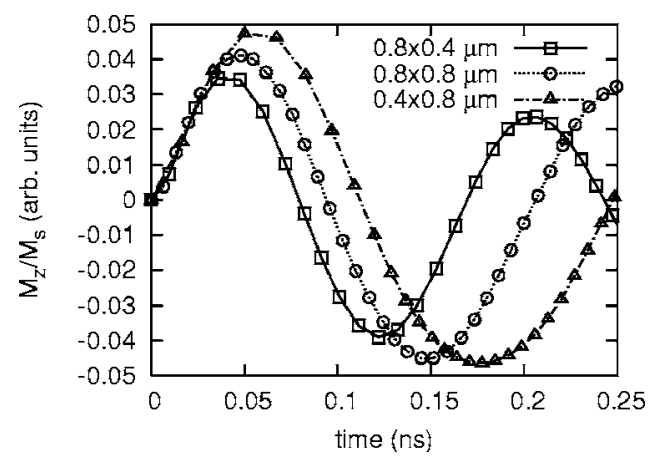

FIG. 4. Simulation of the magnetization dynamics as function of the aspect ratio, showing the higher precession frequency and lower excitation angle for the element $0.8 \times 0.4 \mu \mathrm{m}$ compared to 0.8 $\times 0.8 \mu \mathrm{m}$, and even more to $0.4 \times 0.8 \mu \mathrm{m}$. The applied field $H_{d c}$ was 300 Oe. 


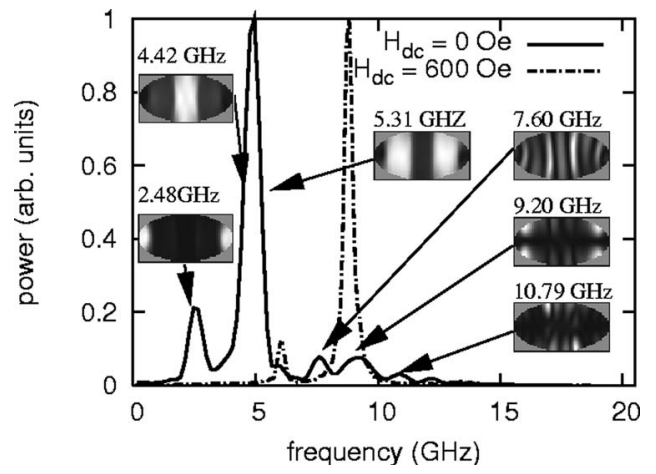

FIG. 5. Simulation of the power spectra and the associated modes for an element size of $400 \times 200 \times 8 \mathrm{~nm}$ for an applied field $H_{d c}$ of 0 Oe and 600 Oe.

higher in that case, resulting in a higher excitation amplitude for elements with $L<W$ than for elements with $L>W$.

For small excitations, the macrospin model approximation for Eq. (1) gives the precession frequency $f \mathrm{as}^{26}$

$$
f=\frac{|\gamma|}{2 \pi} \sqrt{\left|\left[H_{d c}+4 \pi\left(N_{y}-N_{x}\right) M_{s}\right]\left[H_{d c}+4 \pi\left(N_{z}-N_{x}\right) M_{s}\right]\right|},
$$

where $|\gamma|=2.2110^{5} \mathrm{~Hz} \mathrm{~m} / \mathrm{A}$ and $N_{x}, N_{y}$, and $N_{z}$ are the demagnetizing coefficients for a three-dimensional ellipsoid. ${ }^{27}$ The lines in Fig. 3 are calculated in this way and make the link between experiments, which are difficult to realize on small elements, and simulations, which are difficult to realize on large elements.

As mentioned above, only for a real three-dimensional ellipsoid the magnetization dynamics is really homogeneous. For our elliptically shaped thin film elements, the magnetization dynamics is expected to be inhomogeneous and will consist of several modes. These localized modes can be found from the simulations using the following procedure. ${ }^{14}$ First one calculates the power spectrum of each individual cell in which the element has been decomposed for the micromagnetic simulation. For this we used the Lomb-Scargle periodogram, ${ }^{28,29}$ as the time series given by the simulation program are not evenly spaced. One then takes the summation of each of these power spectra over the whole element. This way we prevent some modes from being hidden in the averaged magnetization. Finally, for each of the revealed modes, the spatial distribution of the power at the mode frequency is plotted. This results in a gray-level picture of the element related to the amplitude of the modes, where white and black indicate maximum and zero amplitude, respectively. This way the localization of various modes in the element can be visualized, as shown in Fig. 5.

The parameters we used for the simulation are the same as before, except for the element size which is here $400 \times 200$ $\times 8 \mathrm{~nm}$, and the external applied field $H_{d c}$ which is 0 Oe in this case. The latter is done to pump energy in more modes, in order to make them visible in the spectrum.

All modes present in the element can be classified in three categories. The first one is the main mode, which corresponds to the resonant frequency for the effective field $H_{\text {eff }}$,

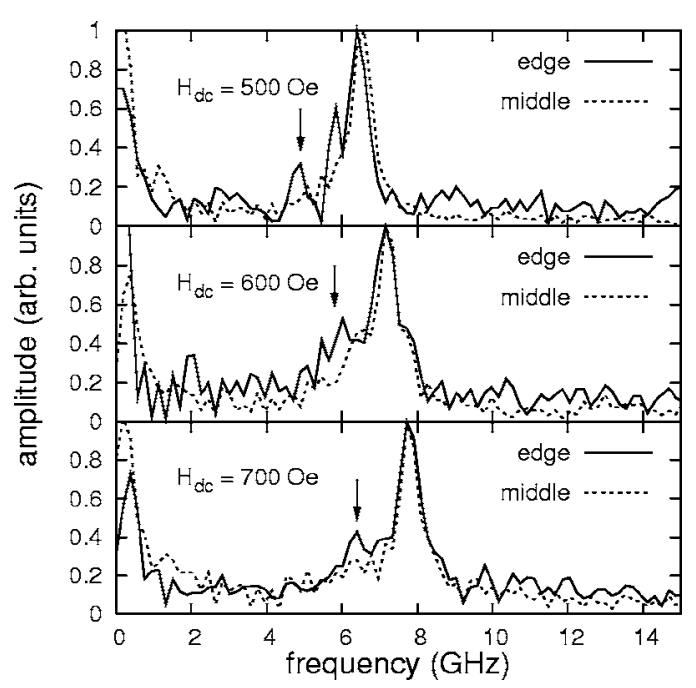

FIG. 6. Measurement of the precession dynamics and resulting power spectra using a pulse field $H_{p}$ of 90 Oe with different applied bias fields $H_{d c}$. Arrows indicate the position of the edge mode.

as already shown in Fig. 3 and follows from Eq. (3). In fact, due to the inhomogeneous demagnetizing field in the element, this main mode is split in two complementary modes, as shown on Fig. 5, with close frequencies at $4.42 \mathrm{GHz}$ and $5.31 \mathrm{GHz}$. The second one is the edge mode, localized at the extreme edges of the element, with a precession frequency of $2.48 \mathrm{GHz}$, as shown in Fig. 5. Finally, there are several modes at higher frequencies, which are highly localized and possess higher symmetry patterns and much less oscillation power than the main modes. These modes are very sensitive to the simulation parameters, like the size of the elements or the applied magnetic field, so at present, it is not possible to relate them to experimental observations.

Because the elements we simulated are much smaller than the ones we measured, we cannot quantitatively compare their spectra. On the other hand, we can qualitatively compare the spectra obtained from our measurements, shown in Fig. 6, with those obtained from the simulations, shown in Fig. 5. In order to resolve the edge mode, we measured both in the middle of the element and on its edge. The power spectra on the edge always show an extra bump close to the main mode but at a lower frequency, which can be identified as the edge mode. This extra bump scales with field, which is in agreement with the simulations. As this extra bump does not appear on the other side of the main modes, it cannot be noise, which would more likely appear at higher frequencies. The decomposition in edge mode, main modes, and higher modes is thus confirmed by our experiments.

An experimental and numerical spectrally resolved study of the magnetization dynamics in a thin ellipsoidal Permalloy element was carried out in order to investigate the effect of the aspect ratio and size of the element on the magnetization dynamics. The presence of localized modes was shown experimentally as well as numerically with micromagnetic simulations. The aspect ratio of the elements was shown to produce a shift in the precession frequency, with stronger effects for smaller elements. Moreover, it was shown experimentally as well as numerically that at least three different 
kinds of localized precession modes exist in such magnetic structures: an edge mode at low frequency, a main mode which can consist of several frequencies close to each other, and higher frequency modes, with a highly localized symmetry. This work is a step towards a more elaborate control of the magnetization switching for MRAM devices, as it high- lights the complexity and nonlinearity of its magnetization dynamics.

We thank the EU projects DYNAMICS, the Dutch organization for Fundamental Research on Matter (FOM), and the NanoNed consortium for financial support.
*Electronic address: 1.leguyader@ science.ru.nl

${ }^{1}$ R. F. Soohoo, Magnetic Thin Films (Harper \& Row, New York, 1965).

${ }^{2}$ C. H. Back, R. Allenspach, W. Weber, S. S. P. Parkin, D. Weller, E. L. Garwin, and H. C. Siegmann, Science 285, 864 (1999).

${ }^{3}$ M. Bauer, J. Fassbender, B. Hillebrands, and R. L. Stamps, Phys. Rev. B 61, 3410 (2000).

${ }^{4}$ J. Miltat, G. Alburquerque, and A. Thiaville, Spin Dynamics in Confined Magnetic Structures (Springer, Berlin, 2001), Vol. 1.

${ }^{5}$ S. Kaka and S. E. Russek, Appl. Phys. Lett. 80, 2958 (2002).

${ }^{6}$ T. M. Crawford, P. Kabos, and T. J. Silva, Appl. Phys. Lett. 76, 2113 (2000).

${ }^{7}$ M. Bauer, R. Lopusnik, J. Fassbender, and B. Hillebrands, Appl. Phys. Lett. 76, 2758 (2000).

${ }^{8}$ Th. Gerrits, H. A. M. van den Berg, J. Hohlfeld, L. Bär, and Th. Rasing, Nature (London) 418, 509 (2002).

${ }^{9}$ H. W. Schumacher, C. Chappert, R. C. Sousa, P. P. Freitas, and J. Miltat, Phys. Rev. Lett. 90, 017204 (2003).

${ }^{10}$ H. W. Schumacher, C. Chappert, P. Crozat, R. C. Sousa, P. P. Freitas, J. Miltat, J. Fassbender, and B. Hillebrands, Phys. Rev. Lett. 90, 017201 (2003).

${ }^{11}$ W. K. Hiebert, G. E. Ballentine, L. Lagae, R. W. Hunt, and M. R. Freeman, J. Appl. Phys. 92, 392 (2002).

${ }^{12}$ A. Krichevsky and M. R. Freeman, J. Appl. Phys. 95, 6601 (2004).

${ }^{13}$ M. Belov, Z. Liu, R. D. Sydora, and M. R. Freeman, Phys. Rev. B
69, 094414 (2004).

${ }^{14}$ R. McMichael and M. Stiles, J. Appl. Phys. 97, $10 J 901$ (2005).

${ }^{15}$ K. Perzlmaier, M. Buess, C. H. Back, V. E. Demidov, B. Hillebrands, and S. O. Demokritov, Phys. Rev. Lett. 94, 057202 (2005).

${ }^{16}$ M. Buess, T. Haug, M. R. Scheinfein, and C. H. Back, Phys. Rev. Lett. 94, 127205 (2005).

${ }^{17}$ G. Gubbiotti, G. Carlotti, T. Okuno, M. Grimsditch, L. Giovannini, F. Montoncello, and F. Nizzoli, Phys. Rev. B 72, 184419 (2005).

${ }^{18}$ T. Devolder and C. Chappert, J. Appl. Phys. 95, 1933 (2004).

${ }^{19}$ H. W. Schumacher, Appl. Phys. Lett. 87, 042504 (2005).

${ }^{20}$ L. Landau and E. Lifshitz, Phys. Z. Sowjetunion 8, 153 (1935).

${ }^{21}$ T. L. Gilbert, Phys. Rev. 100, 1243 (1955).

${ }^{22}$ J. C. Maxwell, Electricity and Magnetism, 3rd ed. (The Clarendon Press, Oxford, 1904), Vol. 2.

${ }^{23}$ M. R. Freeman, M. J. Brady, and J. Smyth, Appl. Phys. Lett. 60, 2555 (1992).

${ }^{24}$ D. V. Berkov and N. L. Gorn, Micromagus-Software for Micromagnetic Simulations, http://www.micromagus.de.

${ }^{25}$ N. Smith, D. Markham, and D. LaTourette, J. Appl. Phys. 65, 4362 (1989).

${ }^{26}$ C. Kittel, Phys. Rev. 73, 155 (1948).

${ }^{27}$ J. A. Osborn, Phys. Rev. 67, 351 (1945).

${ }^{28}$ N. R. Lomb, Astrophys. Space Sci. 39, 447 (1976).

${ }^{29}$ J. D. Scargle, Astrophys. J. 263, 835 (1982). 